\title{
Low mass neutralino dark matter in mSUGRA and more general models in the light of LHC data
}

\author{
Nabanita Bhattacharyya ${ }^{1}$, Arghya Choudhury ${ }^{2}$ and Amitava Datta ${ }^{3}$ \\ Indian Institute of Science Education and Research, Kolkata, \\ Mohanpur Campus, PO: BCKV Campus Main Office, \\ Mohanpur - 741252, Nadia, West Bengal.
}

\begin{abstract}
The $b \tau j \mathbb{E}_{T}$ signal at the ongoing LHC experiments is simulated with Pythia in the mSUGRA and other models of SUSY breaking. Special attention is given on the compatibility of this signature with the low mass neutralino dark matter (LMNDM) scenario consistent with WMAP data. In the mSUGRA model the above signal as well as the LMNDM scenario are strongly disfavored due to the constraints from the on going SUSY searches at the LHC. This tension, however, originates from the model dependent correlations among the parameters in the strong and electroweak sectors of mSUGRA. That there is no serious conflict between the LMNDM scenario and the LHC data is demonstrated by constructing generic phenomenological models such that the strong sector is unconstrained or mildly constrained by the existing LHC data and parameters in the electroweak sector, unrelated to the strong sector, yield DM relic density consistent with the WMAP data. The proposed models, fairly insensitive to the conventional SUSY searches in the jets $+E_{T}$ and other channels, yield observable signal in the suggested channel for $\mathcal{L} \gtrsim 1 \mathrm{fb}^{-1}$ of data. They are also consistent with the LMNDM scenario and can be tested by the direct dark matter search experiments in the near future. Some of these models can be realized by non-universal scalar and gaugino masses at the GUT scale.
\end{abstract}

PACS no:12.60.Jv, 95.35.+d, 13.85.-t, 04.65.+e

\footnotetext{
${ }^{1}$ nabanita@iiserkol.ac.in

2arghyac@iiserkol.ac.in

3adatta@iiserkol.ac.in
} 


\section{Introduction}

Proton - proton collisions at the LHC are now producing data at a center of mass energy $(\sqrt{s})=7 \mathrm{TeV}$. There is no evidence of any new physics beyond the standard model (SM). However, it has already been shown by the ATLAS [1] and CMS [2] collaborations that even with a small integrated luminosity $(\mathcal{L})=35 p b^{-1}$, supersymmetry (SUSY) can be probed much beyond the existing limits on the sparticle masses obtained by the LEP [3] or Tevatron [4] experiments.

The negative results of new particle searches at the LHC have been interpreted in terms of the simplest gravity mediated SUSY breaking model - the minimal supergravity (mSUGRA) [5] model- which has only five free parameters including soft SUSY breaking terms. These are $m_{0}$ (the common scalar mass), $m_{1 / 2}$ (the common gaugino mass), $A_{0}$ (the common trilinear coupling parameter), all given at the gauge coupling unification scale $\left(M_{G} \sim 2 \times 10^{16} \mathrm{GeV}\right)$; the ratio of the Higgs vacuum expectation values at the electroweak scale namely $\tan \beta$ and the sign of $\mu$. The magnitude of $\mu$ is determined by the radiative electroweak symmetry breaking (REWSB) condition [6]. The sparticle spectra and couplings at the electroweak scale are generated by renormalization group evolutions (RGE) of the above soft breaking masses and the coupling parameters. The non-observation of signal, in particular in the jets $+\mathbb{E}_{T}$, channel leads to exclusion plots in the $m_{0}-m_{1 / 2}$ plane. A large number of phenomenological analyses have also addressed the issue of SUSY search at LHC-7 TeV experiments $[7,8]$.

In a hadron collider the dominant source of SUSY signals in the $\mathrm{m} l+\mathrm{n}$ jets $+\mathbb{E}_{T}$ channel is the pair production of the strongly interacting sparticles - the squarks $(\widetilde{q})$ and gluinos $(\widetilde{g})$ - in various combinations. Throughout this paper $l$ stands for $e$ and $\mu$ unless stated otherwise. Thus the bounds from ATLAS [1] and CMS [2] primarily exclude some parameter space with relatively low $m_{0}$ and $m_{1 / 2}$ which translates to certain combinations of squark $\widetilde{q}$ and gluino $\widetilde{g}$ masses ${ }^{4}$. For example, the non-observation of the $0 l+$ jets $+E_{T}$ signal implies that for nearly mass degenerate squarks and gluinos $m_{\widetilde{q}} \approx m_{\widetilde{g}} \geq 775 \mathrm{GeV}$ (see the second paper of [1]). Here $m_{\widetilde{q}}$ stands for the average mass of the $\mathrm{L}$ and $\mathrm{R}$ type squarks.

On the other hand in several regions of the parameter space of the minimal supersymmetric extension of the SM (MSSM) with conserved R-parity but without specific assumptions

\footnotetext{
${ }^{4}$ The constraints become more severe due to the very recent $\mathcal{L}=1 \mathrm{fb}^{-1}$ data as discussed briefly in Section 4 .
} 
about the soft breaking parameters, the dark matter relic density [9, 10] in the universe low mass neutralino dark matter (LMNDM) in particular - essentially depends on the properties of the sparticles in the electroweak (EW) sector. This, e.g., is the case if the lightest neutralino, assumed to be lightest supersymmetric particle (LSP) $\left(\widetilde{\chi}_{1}^{0}\right)$, is bino like and all squarks are beyond the reach of the ongoing LHC experiments.

However, due to the specific correlations among the sparticle masses in mSUGRA, the above bounds on $m_{\widetilde{q}}$ and $m_{\widetilde{g}}$ would also impose stringent indirect mass bounds on the EW sparticles. This disfavors the LMNDM scenario. The bound quoted above from ATLAS data, e.g., implies $m_{\widetilde{l}_{R}} \gtrsim 398$ and $m_{\widetilde{\chi}_{1}^{0}} \gtrsim 125^{5}$. These model dependent bounds imply that the masses of the sparticles belonging to the EW sector are far above the direct lower limits from LEP [3] and too large for realising the LMNDM scenario.

The observed dark matter (DM)relic density $\left(\Omega h^{2}\right)$ in the universe $[9,10]$ has been precisely measured by the Wilkinson Microwave Anisotropy Probe (WMAP) collaboration and is bounded by $0.09 \leq \Omega h^{2} \leq 0.13$ [11]. A possible mechanism of production of relic density in the above range in the LMNDM scenario is annihilation of a bino like LSP pair or bulk annihilation $[10,12,13]$. It may be recalled that, if all strongly interacting sparticles are heavy, relatively low mass neutralinos and $\mathrm{R}$ type sleptons (super partners of $e_{R}, \mu_{R}$ and $\tau_{R}$ ) mainly contribute to this annihilation process. Coannihilation $[13,14]$ of a light neutralino and a nearly degenerate lighter stau mass eigenstate $\left(\widetilde{\tau}_{1}\right)$ is another proposed mechanism for generating the observed relic density. The allowed LMNDM scenarios in the mSUGRA model, with emphasis on the above two processes, have been delineated in the figures in [15] using parameter spaces different from the conventional ones. In this paper we shall frequently refer to these figures. It seems that both the above processes are apparently in conflict with the recent LHC data. The incompatibility of DM relic density production by slepton coannihilation and the data from the LHC-7 TeV experiments have recently been noted in [16].

The indirect 'exclusion' of a light electroweak sector will have a bearing on direct detection of DM [17] as well. The tension between the constraints obtained by the ongoing LHC experiments and the mSUGRA parameter space accessible to direct DM search experiments by the XENON [18] and the CDMS [19] collaborations have also been noted in the literature

\footnotetext{
${ }^{5}$ Throughout this paper all masses, mass parameters and quantities having the dimension of mass are given in $\mathrm{GeV}$ unless stated otherwise.
} 
$[16,20]$. Several groups have also reported on the prospect of constructing the mass of the neutralino by such experiments. It is estimated that if $m_{\widetilde{\chi}_{1}^{0}} \lesssim 150$, then it might be possible to reconstruct this mass by measuring the energy spectrum of the recoiling nuclear targets $[10,21]$. The recoil energy spectrum is insensitive to higher neutralino masses. Moreover the LMNDM scenario can be tested in an $e^{+}-e^{-}$collider if the LSP mass is in the range $1-10$ [22]. However, from the results of direct DM search and/or various constraints from collider and astrophysical experiments it has been claimed that the above mass range is disfavored [23].

In view of the above discussions it is worthwhile to critically reexamine the constraints from the ongoing LHC experiments and their impact on LMNDM scenarios. This will be taken up in a later section. The main conclusion is that in view of the uncertainties in the data, some parameter space with low $m_{0}$ and $m_{1 / 2}$ consistent with LMNDM cannot be conclusively ruled out. However, it must be admitted that there is a tension between the LHC data and the LMNDM scenario in the MSUGRA model.

We remind the reader that before the advent of the bounds from the LHC, the bound $m_{h}>114.4$ on the lighter Higgs scalar mass $\left(m_{h}\right)$ from LEP [24] tightly constrained the parameter space with low $m_{0}-m_{1 / 2}$, a part of which coincides with the parameter space corresponding to the LMNDM scenario. These constraints are particularly severe for low and intermediate values of $\tan \beta[25]$.

It was recently emphasized in $[15,26]$ that in order to revive the parameter space consistent with the LMNDM scenario a moderate to large negative values of the trilinear coupling $\left(A_{0}\right)$ is called for. This is particularly important if $\tan \beta$ is not very large. In fact for $A_{0}$ $=0$ and $\tan \beta=3$ - a choice frequently employed by the LHC and Tevatron experiments - the entire mSUGRA parameter space sensitive to the $35 \mathrm{pb}^{-1}$ data is already excluded by the $m_{h}$ bound. For sizable negative values of $A_{0}$ the LMNDM scenario is realized for another reason. Here the lighter stau mass eigenstate $\left(\widetilde{\tau}_{1}\right)$ becomes significantly lighter than the selectron or the smuon even for moderate $\tan \beta$ and LSP $-\widetilde{\tau}_{1}$ coannihilation may occur efficiently. This happens for a minimum value of $m_{1 / 2}$ much lower than the corresponding value for $A_{0}=0$ (see the figures depicting the parameter space allowed by WMAP data in $[15])$.

This also gives rise to spectacular collider signatures as the lighter chargino $\left(\widetilde{\chi}_{1}^{ \pm}\right)$- or the second lightest neutralino $\left(\widetilde{\chi}_{2}^{0}\right)$ dominantly decay into $\tau$ rich final states. Predictions for 
experiments at LHC-14 TeV [15, 27] and LHC-7 TeV [8] were worked out. In this paper we shall restrict ourselves to non-zero values of $A_{0}$ only.

It is, however, well-known that the $0 l+$ jets $+\mathbb{E}_{T}$ signal is fairly insensitive to the choice of $A_{0}$ and $\tan \beta$ (see the second paper in [1]). Throughout this paper we shall assume that the bounds obtained by the ATLAS and the CMS collaborations in this channel for fixed choices of these two parameters are valid for other choices as well.

Another important consequence of non-zero $A_{0}$ is that the lighter top squark mass eigenstate $\widetilde{t}_{1}$ may be significantly lighter than the other squarks and could be copiously produced at the ongoing LHC experiments. They may come from two dominant sources; i) Direct $\widetilde{t}_{1}-\widetilde{t}_{1}^{*}$ pair production and ii) $\widetilde{g} \rightarrow \widetilde{t}_{1} \bar{t}$, if kinematically allowed.

Our next task is to identify a signal which unlike the jets $+E_{T}$ final state is sensitive to $\left|A_{0}\right|$. If $\widetilde{t}_{1}$ is not the next lightest super particle, then the decay mode $\widetilde{t}_{1} \rightarrow b \widetilde{\chi}_{1}^{+}$may be its main decay channel resulting in final states rich in b-jets. If $\tan \beta$ is small (say, 5) then the electroweak gauginos will dominantly decay leptonically into $e, \mu$ or $\tau$ channels with approximately equal probability. A viable signal in this case would be $\operatorname{blj} \mathbb{E}_{T}$ [28].

For moderate or large $\tan \beta$ and non-zero $A_{0}$ on the other hand the above EW gauginos decay dominantly into final states involving $\tau$ 's leading to very characteristic collider signals $[8,15,27]$. The price to be paid for $\tau$ tagging efficiency may be adequately compensated by the large BRs of the EW gaugino decays into $\tau$ rich final states. The main purpose of this paper is to study the observability $b \tau j E_{T}$ at the ongoing LHC experiments with emphasis on the LMNDM scenario. Here $j$ is the number of jets in the signal and different choices of this variable will be considered. Occasionally, however, we shall also revisit the $b l j E_{T}$ signal [28].

As discussed above there is indeed a tension between the realisation LMNDM in mSUGRA and the preliminary data from the LHC. Should the experimental constraints be strengthened in future, the tension will further intensify. In view of this we propose a few generic models which are either mildly constrained or unconstrained by the current LHC data and are consistent with the low mass neutralino DM scenario. The main point is that the LHC data is sensitive to the masses of the strongly interacting sparticles while the realization of LMNDM in these models DM hinges on the properties of the electroweak sector. Thus if the two sectors are uncorrelated the above tension will cease to exist. These models are generic in the sense that their viability depends on certain mass hierarchies 
among the strongly interacting sparticles rather than on some specific choices of the masses. The important correlations among different mass hierarchies in SUSY models and the corresponding collider signatures have been emphasized in the literature[29]. The parameters in the electroweak sector can be chosen independently. In fact all models where the sparticle masses in the EW sector consistent with the corresponding LEP limits, derived without assuming mSUGRA as the underlying model, are allowed in principle. These models are phenomenological in nature ${ }^{6}$ although we shall comment on theoretical motivations wherever possible. Finally we shall discuss the possibility of testing these models by the ongoing LHC experiments.

The plan of the paper is as follows. In Section 2 we shall mainly concentrate on the phenomenological models unconstrained by the LHC data corresponding to $\mathcal{L}=35 \mathrm{pb}^{-1}$ above and assess the prospect of observing the $b \tau j \quad E_{T}$ signatures. Special emphasis will be given on the LMNDM scenarios. In Section 3 we shall examine the $b \tau j \mathbb{E}_{T}$ signal and occasionally the $b l j E_{T}$ signal in the mSUGRA model using the above data and comment on the viability of realizing the LMNDM scenarios in view of the uncertainties in the LHC constraints. In Section 4 the analyses of Section 2 and 3 are updated in the light of the recent $\mathcal{L}=1 \mathrm{fb}^{-1}$ data. Our conclusions will be summarized in Section 5 .

\section{SUSY signatures and LMNDM in generic models}

The simplest generic model compatible with all LHC data accumulated so far $\left(\mathcal{L}=35 \mathrm{pb}^{-1}\right)$ would be one with all strongly interacting sparticles beyond the reach of $7 \mathrm{TeV}$ experiments while all sparticles in the electroweak sector are light. Unfortunately the earlier simulations in the context of the $14 \mathrm{TeV}$ run indicate that any signature in the current experiments at lower energy is not likely. Thus for a model with non-trivial signatures at this stage of the LHC experiment one needs at least one relatively light strongly interacting sparticle.

In the first model with modest values of $\tan \beta$, only the third generation of squarks and the sparticles in the EW sector are assumed to be within the reach of the early phases of $7 \mathrm{TeV}$ run. The blj $E_{T}$ signal has already been studied in [28] in such a phenomenological

\footnotetext{
${ }^{6}$ In spirit these models are similar to the simplified phenomenological model considered by the ATLAS collaboration with only squarks of the first two generation, the gluinos and the LSP within the reach of the $7 \mathrm{TeV}$ run (see Fig. 2 in the second paper of [1]).
} 
scenario . The large mixing in the top squark mass matrix producing a light mass eigenstate provides a qualitative justification. For small or moderate $\tan \beta$ the b-squark mass eigenstates will be much heavier. Of course a large trilinear soft breaking term $A_{t}$ is needed for the above mixing. It will also yield $m_{h}$ compatible with the LEP bound through radiative corrections.

The current LHC data hardly constrain this model since events from direct low mass $\tilde{t}_{1}-\widetilde{t}_{1}^{*}$ pair production have too little $E_{T}$ or $m_{\text {eff }}$ [28] to survive the strong cuts currently implemented by the ATLAS and CMS experiments for SUSY search. Dedicated searches with softer cuts are called for.

In this model the first two generations of squarks and the gluinos are assumed to be beyond the reach of the early stages of the on going LHC experiments due to some yet unknown soft breaking mechanism. We further assume for the sake of simplicity that $m_{0}$ is the common mass of the squarks belonging to the third generation, the sleptons and the two neutral Higgs bosons. Similarly $m_{1 / 2}$ controls the masses of the electroweak gauginos only. If this partially constrained spectrum yields observable signal over a reasonably large parameter space it is obvious that more will be available in a totally unconstrained MSSM. In fact if the unification of the electroweak gaugino masses at $M_{G}$ is relaxed, the mass of the LSP DM candidate can be even lower [30].

We shall fix $\tan \beta=10$ but take $A_{0}$ as a variable. Again for simplicity the magnitude of $A_{0}$ is restricted to be less than $1 \mathrm{TeV}$. While computing the spectrum we have checked that no charge-colour breaking minimum of the scalar potential [31] occurs. For each point the minimum allowed $A_{0}$ is determined by the $m_{h}$ bound from LEP [24].

The radiative corrections to the lightest Higgs boson mass $\left(m_{h}\right)$ involve some theoretical uncertainties (see, for example, $[15,28]$ for a brief discussion and references to the original works). In view of these uncertainties, if the computed Higgs mass is $m_{h}>110^{7}$ for a point in the parameter space, that point will be regarded as acceptable although the computed mass is somewhat smaller than the direct bound from the direct searches at LEP [24]. Throughout this work the pole mass of the top (bottom) quark will be taken as $m_{t}\left(m_{b}\right)=173(4.25)$ [32]. We shall assume that the masses of the lighter chargino, all the sleptons except the tau mass eigenstates and the third generation squarks are heavier than 100. This is basically a simplified form of the LEP limits. The lighter $\widetilde{\tau}$ mass eigenstate is assumed to be heavier than 82 as required by the LEP data.

\footnotetext{
${ }^{7}$ See footnote 4 .
} 
The light sparticle masses and decay branching ratios (BRs)are generated by SUSPECT [33] and Sdecay [34] and $\mu$ is fixed by the REWSB condition [6].

In the parameter space of interest for the on going sparticle searches at the LHC the two body decays $\widetilde{t}_{1} \rightarrow b \widetilde{\chi}_{1}^{+}$occurs with almost $100 \%$ branching ratio (BR). Moreover in bulk of the parameter space the decays $\widetilde{\chi}_{1}^{+} \rightarrow \widetilde{\tau}_{1} \nu, \quad \widetilde{\nu} \tau$ dominate, leading to the $b \tau j E_{T}$ signature. When the latter decays become subdominant or is kinematically suppressed the decay into a real $\mathrm{W}\left(\widetilde{\chi}_{1}^{+} \rightarrow \widetilde{\chi}_{1}^{0} W\right)$ or a virtual $\mathrm{W}\left(\widetilde{\chi}_{1}^{+} \rightarrow \widetilde{\chi}_{1}^{0} W^{*} \rightarrow \widetilde{\chi}_{1}^{0} f \bar{f}^{\prime}\right)$ open up yielding the $b l j E_{T}$ signature [28].

We have generated $\widetilde{t}_{1} \widetilde{t}_{1}^{*}$ pair events at $E_{C M}=7 \mathrm{TeV}$ using Pythia [35]. The signal $b \tau j E_{T}$ has been simulated using the following procedure. Initial and final state radiation, decay, hadronization, fragmentation and jet formation are implemented following the standard procedures in Pythia.

In this paper all leading order (LO) signal cross-sections have been computed by CalcHEP [36] unless otherwise stated. For any two body final state (except for QCD processes) with identical particles or sparticles both the renormalization and the factorization scales are taken as, $\mu_{R}=\mu_{F}=M$, where $M$ is the mass of the particle or sparticle concerned. For two unequal masses the scales are taken to be the average of the two. For QCD events the scales have been chosen to be equal to $\sqrt{\hat{s}}$ which is the energy in the parton CM frame, and the cross-section is computed by Pythia. All LO cross-sections are computed using CTEQ5L parton density functions (PDFs) [37].

We have considered the backgrounds from $t \bar{t}$, QCD events and $W+n$-jets events, where $W$ decays into all channels. $t \bar{t}$ events are generated using Pythia and the LO cross-section has been taken from CalcHEP which is $85.5 \mathrm{pb}$. QCD processes are generated by Pythia in different $\hat{p}_{T}$ bin : $25 \leq \hat{p}_{T} \leq 400,400 \leq \hat{p}_{T} \leq 1000$ and $1000 \leq \hat{p}_{T} \leq 2000$, where $\hat{p}_{T}$ is defined in the rest frame of the parton collision. The main contribution comes from the low $\hat{p}_{T}$ bin, which has a cross-section of $\sim 7.7 E+07 \mathrm{pb}$. However, for other bins $\left(400<\hat{p}_{T}<1000\right.$ and $1000<\hat{p}_{T}<2000$ ), the background events are negligible.

For $W+n$-jets events we have generated events with $n=0,1$ and 2 at the parton level using ALPGEN (v 2.13) [38]. We have generated these events subjected to the condition that $P_{T}^{j}>20, \Delta R(j, j) \geq 0.3$ and $|\eta| \leq 4.5$. These partonic events have been fed to Pythia for parton showering, hadronization, fragmentation and decays etc.

The next to leading order (NLO) cross-sections for stop-stop pair production have been 
computed by PROSPINO [39] using the CTEQ5M PDFs. The K-factors are computed by comparing with the LO cross-section. The LO cross-sections from PROSPINO agree well with CalcHEP for the same choice of the scales.

The NLO background cross-sections are not known for some backgrounds - in particular for the QCD processes. For computing the significance of the signal we conservatively multiply the total LO background by an overall factor of 2 .

We have used the toy calorimeter simulation (PYCELL) provided in Pythia with the following settings.

- The calorimeter coverage is $|\eta|<4.5$. The segmentation is given by $\Delta \eta \times \Delta \phi=$ $0.09 \times 0.09$ which resembles a generic LHC detector.

- A cone algorithm with $\Delta \mathrm{R}=\sqrt{\Delta \eta^{2}+\Delta \phi^{2}}=0.5$ has been used for jet finding.

- $\mathrm{E}_{\mathrm{T}, \min }^{\mathrm{jet}}=30$ and jets are ordered in $\mathrm{E}_{\mathrm{T}}$.

The signal has been selected as follows:

Lepton Veto:

Leptons $(l=e, \mu)$ are selected with $P_{\mathrm{T}} \geq 10$ and $|\eta|<2.4$. For lepton-jet isolation we require $\Delta R(l, j)>0.5$. For the sake of simplicity the detection efficiency of $e$ and $\mu$ are assumed to be 100\%. Events with isolated leptons are rejected.

b- jet identification:

We have tagged $b$-jets in our analysis by the following procedure. A jet with $|\eta|<2.5$ corresponding to the coverage of tracking detectors matching with a $B$-hadron of decay length $>0.9 \mathrm{~mm}$ has been marked tagged. This criteria ensures that single $b$-jet tagging efficiency (i.e., the ratio of tagged $b$-jets and the number of taggable $b$-jets) $\epsilon_{b} \approx 0.5$ in $t \bar{t}$ events.

$\tau$ - jet identification:

Taus are identified through their hadronic decays producing narrow jets with 1 or 3 tracks pointing to the jets. We have defined a narrow signal cone of size $\Delta R_{S}=0.1$ and an isolation cone of size $\Delta R_{I}=0.4$ around the calorimetric jet axis. We then require 1 or 3 charged tracks inside the signal cone with $\left|\eta_{\text {track }}\right|<2.5$ and $P_{T}>3$ for the hardest track. We further require that there are no other charged tracks with $P_{T}>1$ inside the isolation cone to ensure tracker isolation. 
The following cuts will be call Set 1. These cuts ensure stop rich signal events while rejecting the background efficiently :

- We have selected events with one tagged $b$ jet (cut 1.1).

- We have selected events with one tagged $\tau$ jet (cut 1.2).

- We have rejected events with isolated lepton (cut 1.3).

- Events with missing transverse energy $\left(E_{T}\right) \geq 70$ are selected (cut 1.4).

- We have also demanded events with $P_{T}$ tagged $\tau$ jet $\geq 40$ (cut 1.5).

or

We have demanded events with $P_{T}$ tagged $b$ jet $\leq 50$ (cut $\left.1^{\prime} .5\right)$. This cut is particularly useful if the mass difference between the $\widetilde{t}_{1}$ and the $\widetilde{\chi}_{1}^{ \pm}$is small. It also rejects the $t \bar{t}$ background efficiently.

The set of cuts which includes cut $1^{\prime} .5$ will be referred to as Set $1^{\prime}$.

Our results are summarized in Fig. 1. In the green (online) or small crosshatched (red (online) or big crosshatched)) region the $b \tau j \mathbb{E}_{T}$ signal can be observed for an integrated luminosity of $\mathcal{L}=1(5) \mathrm{fb}^{-1}$. For observability we simply require $S / \sqrt{B}>5$, where $\mathrm{S}$ (B) is the number of signal (background) events. We find that $m_{\widetilde{t}_{1}}$ upto 235 (280) can be probed by this signal with $\mathcal{L}=1(5) \mathrm{fb}^{-1}$.

In the blue (online) or hatched region the decays $\widetilde{\chi}_{1}^{+} \rightarrow \widetilde{\tau}_{1} \nu_{\tau}, \widetilde{\nu}_{\tau} \tau$ are either phase space suppressed or are kinematically forbidden. But the $b l j E_{T}$ signal becomes observable with the cuts of Set 1 proposed in [28] for a suitable $A_{0}$. In the blue (online) or hatched region $m_{\widetilde{t}_{1}}$ varies between 130 - 190 for suitable choices of $A_{0}$. Beyond a certain $m_{0}$ (approximately 480), $A_{0}>1 \mathrm{TeV}$ will be required to make $m_{\widetilde{t}_{1}}$ sufficiently light for an observable signal.

To get a feeling for the relative size of the signal and the SM backgrounds we consider four benchmark points BP1 $(110,170,-540,10)$, BP2 $(110,190,-640,10)$, BP3 $(130,200,-550,10)$, BP4 $(150,230,-745,10)$ which yield observable signals at $\mathcal{L}=1 \mathrm{fb}^{-1}$ (see Fig. 1). The quantities in the bracket are $m_{0}, m_{1 / 2}, A_{0}$ and $\tan \beta$. The stop mass at the four points are 143.6, 165.0, 215.6 and 208.2 respectively. At all four benchmark points $\operatorname{BR}\left(\widetilde{t}_{1} \rightarrow b \widetilde{\chi}_{1}^{+}\right)$and $\operatorname{BR}\left(\widetilde{\chi}_{1}^{+} \rightarrow \widetilde{\tau}_{1}^{+} \nu\right)$ are maximal to a very good approximation. 
The $0 l+$ jets $+E_{T}$ or any other signal which gets contribution from all squark-gluino production is not particularly sensitive to the presence or absence of a light stop. However the $b \tau j E_{T}$ signal proposed here stems from $\widetilde{t}_{1}-\widetilde{t}_{1}^{*}$ pair production alone. Hence it sensitively depends on $m_{\widetilde{t}_{1}}$ and ,consequently, on $A_{0}$. The quoted $A_{0}$ gives the largest signal in each case. However, for each point a range of $A_{0}$ values leads to observable signals. In this range the $\widetilde{t}_{1}-\widetilde{\chi}_{1}^{+}$mass difference corresponding to $\left|A_{0}\right|_{\max }$ is too small for producing a taggable b-jet and the signal becomes weak. In contrast at $\left|A_{0}\right|_{\text {min }}$, the $\widetilde{t}_{1}-\widetilde{t}_{1}^{*}$ production is too suppressed - due to a relatively heavy $\widetilde{t}_{1}$ - to produce a viable signal. This is true for all points in Fig. 1. For example with $m_{0}=90, m_{1 / 2}=180$, the signal is observed in the range $\left|A_{0}\right|=370$ 550. For e.g., $A_{0}=-370,-500$ and -550 observable signals occur for $\mathcal{L}=5 \mathrm{fb}^{-1}, \mathcal{L}=1 \mathrm{fb}^{-1}$ and $\mathcal{L}=5 \mathrm{fb}^{-1}$ respectively.

The LO cross-sections after different cuts for the four benchmark points and different SM backgrounds are presented in Table 1.

The last row gives the significance of the signal for $\mathcal{L}=1 \mathrm{fb}^{-1}$ for the two sets of cuts. The significances, however, are computed on the basis of the NLO cross-sections as discussed above. For the range of $m_{\widetilde{t}_{1}}$ relevant here the K-factor varies rather slowly. We have multiplied the LO signal cross-sections by the average value which is approximately 1.7. As stated above the total SM background is multiplied by an overall factor of 2 .

It follows that larger significance is obtained in some regions by using cut 1.5 while in others cut $1^{\prime} .5$ is more appropriate. The cut 1.5 will be more effective in the region where the $P_{T}^{\tau}$ is harder. This is the case for BP3 as a result of the relatively large mass difference between $\widetilde{\tau}_{1}$ and $\widetilde{\chi}_{1}^{0}$. On the other hand for regions where the difference between $m_{\widetilde{t}_{1}}$ and $m_{\widetilde{\chi}_{1}^{+}}$ is less than 50, cut $1^{\prime} .5$ will be more effective which is the case for BP1, BP2 and BP4.

In Fig. 1 each point marked with a cross is consistent with the DM relic density data ${ }^{8}$. In Figure 1 we also identify the parameter space sensitive to the proposed signal. Therefore, in this reach plot we do not restrict ourselves to points allowed by the WMAP data only. In fact at many points in the delineated parameter space the computed $\Omega h^{2}$ violates even the WMAP upper bound on the relic density. If a signal is indeed observed at any of these points, it would indicate that the lightest neutralino though stable in the time scale of a

\footnotetext{
${ }^{8}$ We remind the reader that the parameters $m_{0}$ and $m_{\frac{1}{2}}$ used here are different from the common scalar and gaugino masses in mSUGRA. We have defined these parameters in the present context in Section 2, paragraph 4 .
} 


\begin{tabular}{|c|c|c|c|c||c|c|c|c|}
\hline & \multicolumn{5}{|c|}{ Signal } & \multicolumn{4}{c|}{ Background } \\
\hline & BP1 & BP2 & BP3 & BP 4 & $t \bar{t}$ & QCD & $W+1 j$ & $W+2 j$ \\
& 38.9 & 19.2 & 4.56 & 5.54 & 85.5 & $7.7 \times 10^{7}$ & $1.43 \times 10^{4}$ & 5200 \\
\hline \hline cut 1.1 & 4.6251 & 3.5190 & 2.2193 & 1.6781 & 42.1780 & $6.454 \times 10^{5}$ & 44.0797 & 48.1231 \\
\hline cut 1.2 & 1.0696 & 0.6797 & 0.7327 & 0.5540 & 3.8987 & $6.426 \times 10^{4}$ & 2.7408 & 2.9982 \\
\hline cut 1.3 & 0.8581 & 0.5443 & 0.5365 & 0.4164 & 2.8266 & $6.348 \times 10^{4}$ & 2.4310 & 2.6816 \\
\hline cut 1.4 & 0.2899 & 0.1887 & 0.2469 & 0.1689 & 0.7301 & 22.1066 & 0.1787 & 0.3067 \\
\hline cut 1.5 & 0.2075 & 0.1262 & 0.1802 & 0.1278 & 0.5318 & 0.0102 & 0.1191 & 0.2126 \\
or & & & & & & & & \\
cut 1'.5 & 0.2067 & 0.1273 & 0.0784 & 0.1045 & 0.1855 & 0.0001 & 0.0595 & 0.0940 \\
\hline S/ $\sqrt{B}$ & & & & & & & & \\
After cut 1.5 & 8.4 & 5.1 & 7.3 & 5.2 & & & & \\
After cut 1'.5 & 13.4 & 8.3 & 5.1 & 6.8 & & & & \\
\hline
\end{tabular}

Table 1: The LO cross-sections (including efficiency) for the signal corresponding to BP1 BP4 in the phenomenological model ( section 2, paragraphs 2 - 4) and the SM backgrounds after Set 1 or Set $1^{\prime}$ of cuts. The last row gives the significance of the signal for $\mathcal{L}=1 \mathrm{fb}^{-1}$ on the basis of NLO cross-sections (see text).

collider event may be cosmologically unstable. A tiny R-parity violation induced by higher dimensional operators, for example, may induce neutralino decays at the cosmological scale. In such cases the observed relic density must come from some other non-neutralino sources.

In [15] it was shown that there are two generic regions corresponding to LMNDM. There is a region where neutralino pair annihilation via R-type light slepton exchange or bulk annihilation produces the observed relic density. The tension between the computed lighter Higgs scalar mass $\left(m_{h}\right)$ and the corresponding experimental lower bound is softened by nonzero but moderate negative values of $A_{0}(200-300)$ and the uncertainties in the computed $m_{h}$. Here the $b \tau j E_{T}$ signal is observable since the $\widetilde{\tau}_{1}-\widetilde{\chi}_{1}^{0}$ mass difference is relatively large. Hence taggable $\tau$-jets arise from the decay $\widetilde{\tau}_{1} \rightarrow \tau \widetilde{\chi}_{1}^{0}$.

In the other regions consistent with LMNDM the lighter $\widetilde{\tau}$ mass eigenstate is much lighter due to larger values of $\left|A_{0}\right|(\mathcal{O}(1 \mathrm{TEV}))$. As a result $\widetilde{\tau}_{1}-\widetilde{\chi}_{1}^{0}$ coannihilation along with bulk 


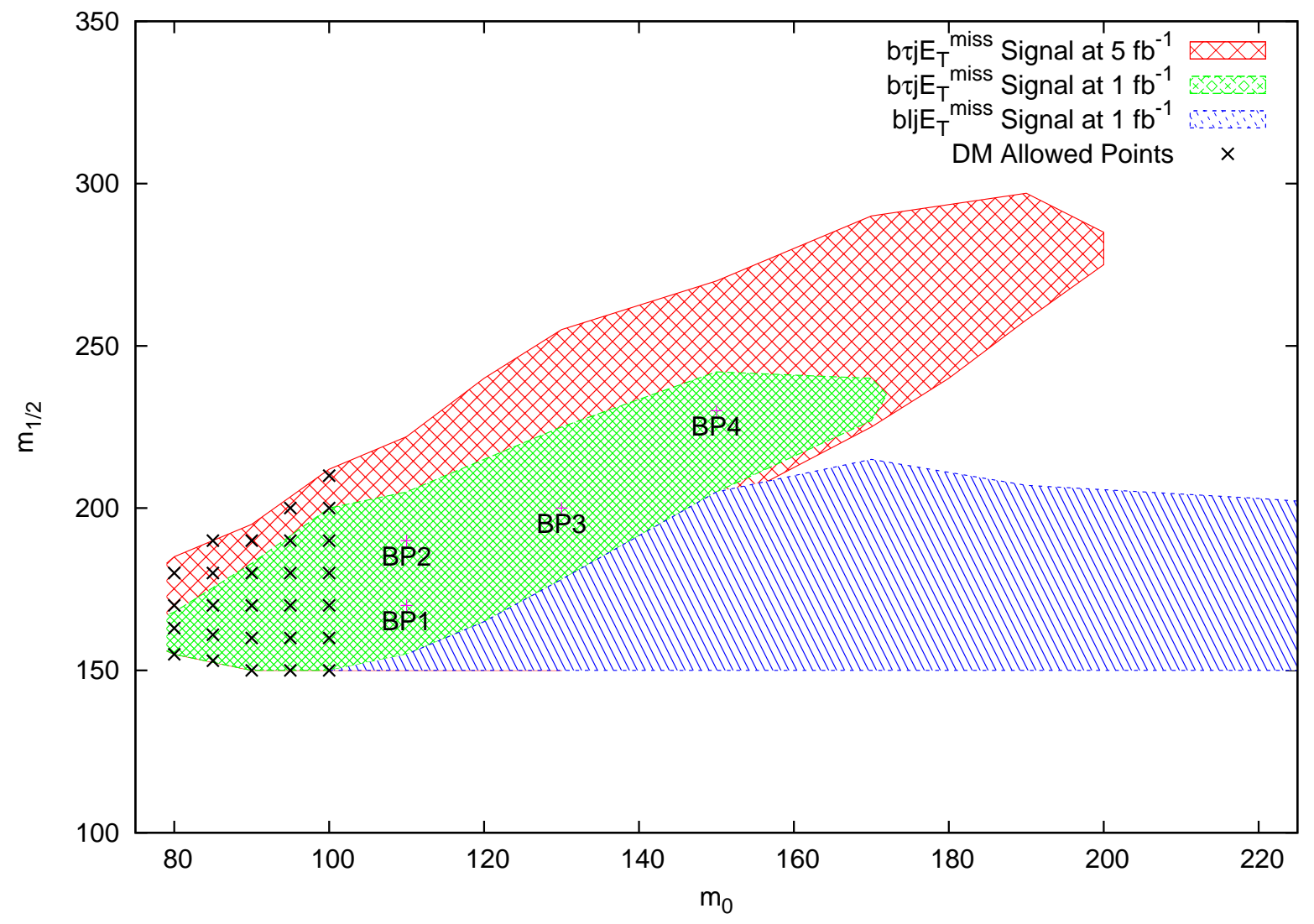

Figure 1: Regions of $m_{0}-m_{1 / 2}$ plane which can be probed by direct production of $\widetilde{t}_{1} \widetilde{t}_{1}^{*}$ pairs for $1 \leq \mathcal{L} \leq 5 \mathrm{fb}^{-1}$ (see Section 2, paragraph $2-4$ for the details of the underlying phenomenological model). The SM backgrounds are in Table 1.

annihialion produce the LMNDM. However, due to the small $\widetilde{\tau}_{1}$ - LSP mass difference the $b \tau j E_{T}$ signal from stop pair production may not be viable.

The next scenario we consider is one with two strongly interacting sparticles within the reach of the first phase of the LHC experiment along with electroweak sparticles. It is assumed that the production of the lighter top squark and the gluinos is the primary source of the LHC signatures. The other strongly interacting sparticles are assumed to be beyond the reach of the LHC-7 TeV run. The masses of all strongly interacting sparticles are taken to be independent of the electroweak sector. For simplicity the masses of the sparticles and 
other relevant parameters in the EW sector are chosen as in mSUGRA. One is thus free to choose any LMNDM scenario. This will be referred to as the Light Stop Gluino (LSG) scenario.

We shall first consider a phenomenological model with $m_{\widetilde{t}_{1}}$ and $m_{\widetilde{g}}$ as unrelated parameters at the weak scale. We shall also consider a mSUGRA type scenario with non-universal boundary conditions at the GUT scale which leads to the LSG scenario.

However, the parameter space in the LSG scenario is already constrained to some extent by the $35 \mathrm{pb}^{-1}$ LHC data. The ATLAS collaboration has analysed the jets $+\mathbb{E}_{T}$ signal using four sets of cuts referred to as A, B, C, D [1]. The corresponding lower limits on the production cross-sections of all strongly interacting sparticles including the efficiencies are 1.3, $0.35,1.1$ and $0.11 \mathrm{pb}$ respectively. These limits are converted into constraints in the $m_{0}-m_{1 / 2}$ plane by computing the NLO cross-section by PROSPINO and the efficiencies by Pythia.

In mSUGRA the set D consisting of very hard cuts is very potent for obtaining new mass bounds. In the LSG scenario, however, this set kills the signal in any LSG model consistent with the Tevatron bounds on $m_{\widetilde{g}}$ for heavy squarks [4]. When each point in the parameter space is required to pass all the above cut sets, set $\mathrm{A}$ and set $\mathrm{C}$ turns out to be most effective in obtaining limits in the LSG scenario. The resulting bounds are $m_{\widetilde{g}} \geq 320-330$ for $m_{\widetilde{t}_{1}}=$ 150 - 300. The insensitivity of the bound on $m_{\widetilde{g}}$ to $m_{\widetilde{t}_{1}}$ clearly indicates that the hard cuts employed by the ATLAS group in isolating the jets $+E_{T}$ signal eliminates all events from stop pair production. The bound essentially comes from gluino pair production followed by the decay of each gluino into top-stop pairs. Hence the canonical signals with strong cuts are not suitable for revealing the presence of a light stop. Interesting contribution to the signal may, however, originate from gluino decays.

For computing the above limits we have set the masses of the squarks (also sleptons) of $\mathrm{L}$ and $\mathrm{R}$ type belonging to the first two generations at $1.5 \mathrm{TeV}$. We have treated $m_{\widetilde{t}_{L}}, m_{\widetilde{t}_{R}}, m_{\widetilde{b}_{R}}, A_{t}, A_{b}$ as well as $m_{\widetilde{g}}$ as free parameters. We have chosen the following parameters for the electroweak sector at the weak scale as $M_{1}=60, M_{2}=125, m_{\widetilde{\tau}_{L}}=155, m_{\widetilde{\tau}_{R}}=116$, $A_{\tau}=-615, \tan \beta=10, \mu=348$. This choice of parameters gives $m_{\widetilde{\chi}^{ \pm}}=122, m_{\widetilde{\chi}_{1}^{0}}=59$, $m_{\widetilde{\tau}_{1}}=109, m_{\widetilde{\nu}_{\tau_{1}}}=142$ and compatible with the WMAP data. The above limits mildly depend on the variation of the parameters in the EW sector unless one makes very specific choices such that the decay pattern of the EW gauginos are drastically different ( e.g., they 
all decay leptonically with 100\% BR).

SUSY searches at the Tevatron can also potentially constrain the LSG scenario. We have not done the analysis in this paper. As a cautious approach we have only considered $m_{\widetilde{g}} \gtrsim$ 400 which corresponds to the bound on $m_{\widetilde{g}}$ if all squarks are heavy and is stronger than the limits discussed above.

We next compute the $b \tau j E_{T}$ signal for different $m_{\widetilde{t}_{1}}$ and $m_{\widetilde{g}}$ in the LSG scenario. The other parameters are fixed as follows: $m_{\tilde{q}_{L / R}(1,2)}=m_{\widetilde{l}_{L}}=m_{\widetilde{l}_{R}}=1.5 \mathrm{TeV}, m_{\widetilde{\chi}_{1}^{ \pm}}=152.6, m_{\widetilde{\chi}_{1}^{0}}$ $=79.1, m_{\widetilde{\tau}_{1}}=144.1$. We take wino like $\tilde{\chi}_{1}^{ \pm}$and bino like $\tilde{\chi}_{1}^{0}$. The electroweak sector is chosen such that the resulting DM relic density is consistent with WMAP data.

\begin{tabular}{|c|c|c|c||c|c|c|}
\hline Points & \multicolumn{3}{|c|}{$\widetilde{t}_{1} \widetilde{t}_{1}^{*}$} & \multicolumn{3}{c|}{ LSG scenario } \\
\hline$m_{\widetilde{t}_{1}}-m_{\widetilde{g}}$ & Cut Set 1 & Cut Set 2 & Cut Set 3 & Cut Set 1 & Cut Set 2 & Cut Set 3 \\
\hline $209-504$ & $359.0(8.6)$ & $270.3(8.8)$ & $3.8(0.9)$ & $463.4(11.1)$ & $280.5(9.1)$ & $28.7(7.2)$ \\
\hline $250-443$ & $204\left(4.9^{* *}\right)$ & $136.7\left(4.4^{* *}\right)$ & $6.8(1.7)$ & $285.3(6.8)$ & $144.7\left(4.7^{* *}\right)$ & $25.4(6.3)$ \\
\hline $250-517$ & $209(5.0)$ & $140.7\left(4.5^{* *}\right)$ & $6.6(1.6)$ & $239.2(5.7)$ & $139.2\left(4.5^{* *}\right)$ & $20.1(5.0)$ \\
\hline
\end{tabular}

Table 2: Number of $b \tau j E_{T}$ events from pure $\widetilde{t}_{1} \widetilde{t}_{1}^{*}$ production (columns $2-4$ ) and that in the phenomenological LSG scenario (columns 5 - 7) for three sets of cuts (see text). Numbers in the brackets are the significance of the signal for $\mathcal{L}=1 \mathrm{fb}^{-1}$ using NLO cross-section and the entries marked with $* *$ indicate that the signal is observable for $1<\mathcal{L} \leq 5 \mathrm{fb}^{-1}$.

We analyse the signal with 3 sets of cuts and the results are summarized in table 2 . The Cut Set 1 and the corresponding SM background have already been given. Both stop and gluino production can contribute to the signal. If the stop is relatively heavy and the gluino is relatively light the size of the overall BSM signal may improve considerably to the case where only $\widetilde{t}_{1}$ of the same mass is present.

The Cut Set 2 has two cuts in addition to Cut Set 1:

- $N_{\text {Central-jet }} \leq 4$ ( where the central jets have $|\eta| \leq 2.5$ ) .

- $M_{\text {eff }} \leq 500$ ( where $\left.M_{\text {eff }}=\left|\mathbb{F}_{T}\right|+\Sigma_{i}\left|P_{T}^{j_{i}}\right|+\Sigma_{i}\left|P_{T}^{l_{i}}\right|\left(l_{i}=e, \mu\right)\right)$.

The corresponding SM background is $0.4744 \mathrm{pb}$. After this set of cuts the gluino induced events in the sample are drastically reduced. As discussed in [28] this sample can be used 
for studying the properties of the light stop squarks. Moreover, if the signal comes from stop pair production alone the fraction of events surviving this cut is much larger than that if the signal stems from both $\widetilde{t}_{1}$ and gluino induced events. This distinction, however, becomes unclear as $m_{\widetilde{g}}$ increases.

The Cut Set 3 is designed to remove the pure stop induced events. It consists of the following stronger cuts :

- We have selected events with one tagged $b$ jet (cut 3.1).

- We have selected events with one tagged $\tau$ jet (cut 3.2).

- We have rejected events with isolated leptons (cut 3.3).

- Events with at least 6 central jets are selected, where central jets are defined as pycell jets with $|\eta| \leq 2.5$ (cut 3.4).

- Events with missing transverse energy $\left(E_{T}\right) \geq 160$ are selected (cut 3.5).

\begin{tabular}{|c|c|c|c|c|}
\hline & $t \bar{t}$ & $\mathrm{QCD}$ & $W+1 j$ & $W+2 j$ \\
\hline cut 3.1 & 42.1780 & $6.454 \times 10^{5}$ & 44.0797 & 48.1231 \\
\hline cut 3.2 & 3.8987 & $6.426 \times 10^{4}$ & 2.7408 & 2.9982 \\
\hline cut 3.3 & 2.8266 & $6.348 \times 10^{4}$ & 2.4310 & 2.6816 \\
\hline cut 3.4 & 0.4454 & 0.0256 & 0.0476 & 0.2275 \\
\hline cut 3.5 & 0.0076 & 0.0004 & - & - \\
\hline
\end{tabular}

Table 3: The LO cross-section (including efficiency) of SM backgrounds after the Set 3 of cuts.

Only The gluino induced events survive and depending on the gluino mass may give an observable signal. The significance of the signal for $\mathcal{L}=1 \mathrm{fb}^{-1}$ for each case is given in parentheses in Table 2. The corresponding SM backgrounds are presented in Table 3. For the gluino mass range in Table 2 the $\mathrm{K}$ factor varies between 1.6 and 1.7.

The LSG scenario can be realized in gravity mediated SUSY breaking with non-universal masses at $M_{G}$. All scalar superpartners squarks belonging to the first two generations are 
assumed to have masses beyond the reach of the ongoing runs at the LHC. The squarks belonging to the third generation are assumed to have much smaller masses. At $M_{G}$ the $\widetilde{\tau}$ mass is assumed to be even smaller so that the LMNDM can be eventually realized. Qualitatively the non-universal masses for the third generation can be generated by the running of the corresponding soft breaking parameters, from the SUSY breaking scale down to $M_{G}$ [40], by effective mass terms induced at $M_{G}$ by some yet unknown flavour dependent interactions above the GUT scale etc, etc. The phenomenology of these models has attained due attention [41].

The gluino mass $\left(M_{3}\right)$ is assumed to be smaller than the other gaugino masses at $M_{G}$. For simplicity it is assumed that $M_{1}=M_{2}$ at this scale. They are chosen such that they are compatible with a LMNDM scenario. The motivation for non-universal gaugino masses have already been discussed by several authors [42]. It arises if a GUT nonsinglet chiral superfield couples to the gauge kinetic function and the hierarchy among the non-universal gaugino masses depends on the representations of the GUT group to which the chiral superfield belongs. In practice linear combinations of such chiral superfields may couple, making the prediction of the above mass hierarchy rather difficult. The phenomenology non-universal gaugino masses has also been discussed extensively [43]. Models with both the above nonuniversalities have also been constructed [44] and the resulting phenomenology were analyzed $[45]$.

Finally in order to get the magnitude of $\mu$ consistent with a LMNDM scenario with bino like LSP, we have to introduce non-universal soft breaking Higgs masses $m_{H_{u}}$ and $m_{H_{d}}$ at $M_{G}[46]$.

For illustration we have chosen the following spectrum at $M_{G}$ which is consistent with a LMNDM scenario satisfying the WMAP constraints, where the relic density is produced by stau - LSP coannihilation: $m_{0}(1,2)=1.5 \mathrm{TeV}, m_{H_{u}}=300, m_{H_{d}}=500, M_{1}=M_{2}=240$, $A_{t}=A_{b}=A_{\tau}=-500, \tan \beta=10$.

The parameter $m_{0}(3)(\widetilde{t})$, the common mass for the third generation of squarks $\left(\widetilde{Q}_{L}, \widetilde{t}_{R}\right.$, $\left.\widetilde{b}_{R}\right)$ and the gluino mass $M_{3}$ at $M_{G}$ are the variables. The common mass $m_{0}(3)(\widetilde{\tau})$ of the third generation of sleptons $\left(\widetilde{\tau}_{L}, \widetilde{\tau}_{R}, \widetilde{\nu}_{\tau}\right)$ is mildly varied in a small range to obtain a LMNDM scenario. The variation of the soft breaking terms at $M_{G}$ and the resulting physical masses of the relevant sparticles are presented in Table 4 .

In the phenomenological LSG scenario $m_{\widetilde{t}_{1}}$ is a weak scale input and $A_{t}$ is not important. 


\begin{tabular}{|c|c|c||c|c|c|c|c||c||c|}
\hline$M_{3}$ & $m_{0}(3)$ & $m_{0}(3)$ & $m_{\widetilde{t}_{1}}$ & $m_{\widetilde{\chi}_{1}^{ \pm}}$ & $m_{\widetilde{\tau}_{1}}$ & $m_{\widetilde{\chi}_{1}^{0}}$ & $m_{\widetilde{g}}$ & $\begin{array}{c}\text { S1 } \\
(\text { Cut Set 1) }\end{array}$ & $\begin{array}{c}\text { S3 } \\
(\text { Cut Set 3) }\end{array}$ \\
\hline \multirow{4}{*}{150} & 460 & 112 & 214 & 187 & 106.3 & 98.0 & 429 & $329.8(7.9)$ & $35.2(8.8)$ \\
& 520 & 117 & 288 & 190 & 107.4 & 98.6 & 435 & $196.3\left(4.7^{* *}\right)$ & $20.1(5.0)$ \\
& 580 & 121 & 351 & 192 & 107.7 & 98.9 & 438 & $136.1\left(3.3^{* *}\right)$ & $18.7\left(4.7^{* *}\right)$ \\
\hline 170 & 460 & 114 & 250 & 188 & 106.6 & 98.0 & 475 & $235.6(5.6)$ & $23.6(5.9)$ \\
\hline 200 & 460 & 117 & 302 & 189 & 107.4 & 98.0 & 547 & $75.8(1.8)$ & $10.6\left(2.6^{* *}\right)$ \\
\hline
\end{tabular}

Table 4: Some representative non-universal input parameters at $M_{G}$ leading to the LSG scenario and the corresponding sparticle spectra. The EW sector yields DM relic density consistent with WMAP data. Last two columns give number of $b \tau j E_{T}$ events (using NLO cross-sections) under cut Set 1 and Set 3 for $\mathcal{L}=1 \mathrm{fb}^{-1}$. Significance for each case is given in the bracket and entries marked with ${ }^{* *}$ indicate that signal is observable for $1<\mathcal{L} \leq 5$ $\mathrm{fb}^{-1}$.

In the LSG scenario obtained from non-universal GUT scale boundary conditions, a smaller $m_{\widetilde{t}_{1}}$ contributes more to the size of the $b \tau j E_{T}$ signal obtained with softer cuts (see the last but one columns of table 4 and section 4 ). Hence $A_{t}$ at $M_{G}$ is an important parameter (see the inputs for table 4).

The prospect of observing the $b \tau j E_{T}$ signal at the ongoing LHC experiments in the above LSG scenario is also summarized in Table 4 . It follows that for a fixed $M_{3}=150\left(m_{\tilde{g}} \approx 435\right)$, $m_{\widetilde{t}_{1}} \leq 350$ can be probed with $\mathcal{L}$ a little more than $1 \mathrm{fb}^{-1}$.

The $b \tau j E_{T}$ signal, which arises when the chargino decays into $\tau$ rich final states with large BR, has not so far been searched by the LHC collaborations. The ATLAS collaboration has already searched for the $b l j E_{T}$ signal arising from the decay of stop pairs using the 35 $\mathrm{pb}^{-1}$ data (see the third paper in ref. 1). Such pairs may either be produced directly or via two body decay of gluino pairs produced at the LHC. Apparently the experiment takes into account both possibilities and excludes $m_{\widetilde{g}}$ below $530-540$ for $m_{\widetilde{t}_{1}}=125-300$. However, the insensitivity of the gluino mass limit on $m_{\widetilde{t}_{1}}$ indicates that the events from directly produced stop pairs are eliminated by the selection procedure and effectively their limits arise from the gluino induced processes. As already shown in [28] and also in this paper directly produced 
light top squarks can also contribute to the signal under a different search strategy based on softer cuts. The possibility that only lighter stop squark pairs have been produced at the LHC yielding either the $b l j E_{T}$ or $b \tau j E_{T}$ signature still remains open.

We first employ relatively soft cuts so that the search is sensitive to both direct stop pair production and gluino pair production followed by the decay of each gluino into top-stop pairs. This enhances the size of the overall SUSY signal especially for relatively heavy stop $\left(m_{\widetilde{t}_{1}} \geq 250\right)$ and light gluino. As demonstrated above one can design cuts which can separate the signal stemming from the two different production channels.

As already noted the simplest model practically unconstrained by the LHC data would of course be the one in which all strongly interacting sparticles are beyond the reach of the LHC experiments at $7 \mathrm{TeV}$. This model can be motivated by introducing non-universal gaugino masses in a mSUGRA like framework by requiring $M_{3}>>m_{1 / 2}, m_{0}$ at $M_{G}$, where $M_{3}$ is the gluino mass and $m_{1 / 2}$ is the common mass of the $S U(2)$ and $U(1)$ gauginos [47]. The rest of the sparticle masses may be determined by mSUGRA like parameters $m_{0}, M_{1}=M_{2}=m_{1 / 2}, A_{0}, \tan \beta$ and $\operatorname{sign}(\mu)$. The parameter $m_{0}$, assumed to be much smaller than $M_{3}$ at $M_{G}$, controls the scalar masses in the electroweak sector but has very little impact on the masses of the strongly interacting sparticles. The latter parameters can be chosen such that they are consistent with the LMNDM scenario.

Unfortunately the signal will not be easy to observe at the on going LHC experiments. The slepton pair production [48] and the clean trilepton signal [49] from chargino $\left(\widetilde{\chi}_{1}^{ \pm}\right)$-second neutralino $\left(\widetilde{\chi}_{2}^{0}\right)$ pair production would be the most distinctive signatures of this model. It is, however, well known that even for the $14 \mathrm{TeV}$ experiment the reach in the trilepton channel is rather modest [50]. This does not suggest an exciting prospect for the $7 \mathrm{TeV}$ run. At 14 $\mathrm{TeV}$ the reach can be improved by including the $1 l+2 \tau$ or $2 l+1 \tau$ events arising from the decays of $\tilde{\chi}_{1}^{ \pm}$and $\tilde{\chi}_{2}^{0}$ [47]. The reach for slepton search is also rather limited at $14 \mathrm{TeV}$ [51]

These signals, however, have not been studied for the on going run nor is the prospect of slepton search at $7 \mathrm{TeV}$ known. In this paper we did not pursue this model any further. 


\section{SUSY signatures and LMNDM in mSUGRA in view of LHC data with $\mathcal{L}=35 \mathrm{pb}^{-1}$}

In this section we consider the prospect of observing the $b \tau j E_{T}$ signal at LHC-7 TeV and realizing the LMNDM scenario in mSUGRA. However, the ATLAS collaboration has obtained strong constraints on the mSUGRA parameter space [1] which should be taken into account. We remind the reader that the ATLAS results were obtained for $A_{0}=0$ and $\tan \beta=3$ - a choice forbidden by the direct bound on $m_{h}$ from LEP. However, in the same paper they noted that the limits are rather insensitive to the variation of the above two parameters. We shall, therefore, assume that these limits are valid in more general models with different $A_{0}$ and $\tan \beta$ values.

Our results are summarized in Fig. 2. This figure corresponds to $A_{0}=-600$ and $\tan \beta=$ 10. The observed ATLAS limits (reproduced in Fig. 2) are rather strong and strongly disfavors the LMNDM scenario. However, these limits were obtained with the strongest set of cuts used by them (signal region D in [1]) resulting in 2 observed events against a SM expectation of $2.5 \pm 1.0_{-0.4}^{1.0} \pm 0.2$. An upward fluctuation of the observed number, which is certainly a distinct possibility, would relax the limits significantly. Thus the variation of the expected limit, which is very close to the observed limit in this case, in response to $\pm 1 \sigma$ fluctuation of the SM expectation is perhaps a more realistic description of our present knowledge. The band resulting from the above fluctuations as given in [1] is also included in Fig. 2.

We also present the points (denoted by the $\mathrm{x}$ mark) in the low $m_{0}-m_{1 / 2}$ region allowed by the DM relic density data. The relic density is mainly produced by stau-LSP coannihilation with some contribution from bulk annihilation. The observed limits disfavor most of the region consistent with the LMNDM scenario. In fact $m_{\widetilde{\chi}_{1}^{0}} \leq 143$ is disfavored. However, if the uncertainties in the limits are taken into account $\widetilde{\chi}_{1}^{0}$ with smaller masses can not be the excluded with certainty as DM candidates. It is also to be noted that the computed $m_{h}$ for $A_{0}=0$ also puts pressure on the low $m_{0}-m_{1 / 2}$ region even after theoretical uncertainties in the computation is taken into account.

The Cut Set 3 introduced in Section 2 is used for estimating the signal and the background (see Table 3). In the green (online) or small crosshatched region the signal is observable with $\mathcal{L}=1 \mathrm{fb}^{-1}$. No signal from the points consistent with the observed relic density is 


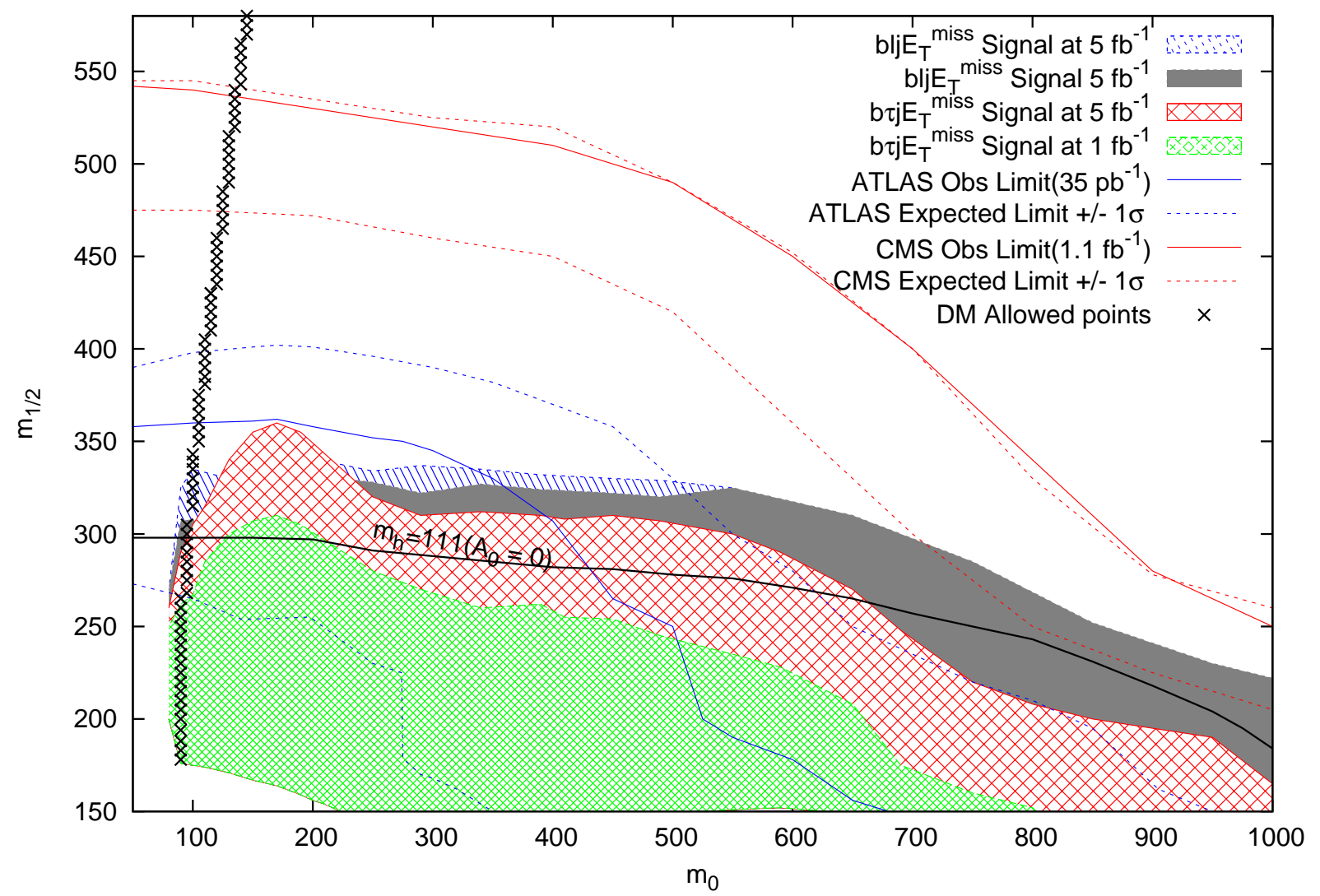

Figure 2: The green (online) or small crosshatched (red (online) or big crosshatched)) region of $m_{0}-m_{1 / 2}$ plane in mSUGRA can be probed by the $b \tau j E_{T}$ signal from all squark-gluino events using Set 3 of cuts with $\mathcal{L}=1(5) \mathrm{fb}^{-1}$. The $b l j \mathbb{E}_{T}$ signal probes the grey (blue (online) or hatched) region with the cuts proposed in [28] (cut Set 4 introduced in this paper) for $1<\mathcal{L} \leq 5 \mathrm{fb}^{-1}$. Here $A_{0}=-600$ and $\tan \beta=10$, $\operatorname{sign}(\mu)>0$ (see text for the details).

expected for DM allowed points even after considering the uncertainties in the ATLAS data. For $1 \mathrm{fb}^{-1}<\mathcal{L}<5 \mathrm{fb}^{-1}$ the signal is observable over a much larger region of the parameter space (the red (online) or big crosshatched region). A few points allowed by the relic density data yield observable signals (see fig. 2).

We next comment briefly on the $b l j E_{T}$ signal. In addition to the Set 3 of cuts defined 
in [28] we introduce a new set of cuts (Set 4) given below.

- We have selected events with one tagged $b$ jet.

- We have selected events with one isolated lepton .

- Events with at least 6 central jets are selected, where central jets are defined as pycell jets with $|\eta| \leq 2.5$.

- Events with missing transverse energy $\left(E_{T}\right) \geq 160$ are selected.

The former set gives a better reach in $m_{1 / 2}$ for $m_{0}<550$ while the second set is more effective for larger $m_{0}$ for $\mathcal{L}=5 \mathrm{fb}^{-1}$. This is illustrated in Fig. 2 by the grey and the blue (online) or hatched regions respectively.

Recently several groups have reexamined the sensitivity of the on going XENON100 experiment in the light of the constraints on the mSUGRA model imposed by the LHC $35 \mathrm{pb}^{-1}$ data. The Figure 2(L) of [20], for example, indicates that the parameter space sensitive to the above experiment is not compatible with the LHC constraints. However, if the sensitivity of XENON experiment is improved by a factor of 10, which may be achieved by the end of 2012, a much larger region of the mSUGRA parameter space may be accessible to direct search experiments. Discovery of neutralino DM by direct search in the near future would, therefore, suggest models beyond mSUGRA like the ones discussed in this paper.

\section{The generic models, the mSUGRA model and the LMNDM scenario revisited in view of the $\mathcal{L}=1 \mathrm{fb}^{-1}$}

\section{data}

After submitting this paper to the arXiv and the journal. the constraints on the mSUGRA model from LHC data with $\mathcal{L}=1 \mathrm{fb}^{-1}$ were announced. In this section we discuss briefly the impact of the new data on our main results. The following discussion is based on the results presented in the Lepton-Photon conference, 2011 [52] and in the CMS paper [53].

Of course the results for the $b \tau j E_{T}$ signal from stop pair production alone in the first part of Section 2 remain unaltered. As already discussed, none of the CMS and ATLAS 
searches employing very hard $E_{T}$ cuts are sensitive to either the $b l j E_{T}$ signal discussed in [28] ${ }^{9}$ or the $b \tau j E_{T}$ channel discussed in this paper. A dedicated search for relatively low mass top squarks using softer cuts as out lined in Section 2 is called for. Also the search for final states with one tagged b-jet and one $\tau$ jet has not been reported so far by the LHC collaborations.

The stronger constraints from the new data in the jets $+E_{T}$ channel will increase the lower bounds on $m_{\widetilde{g}}$ in the LSG scenario presented in Section 2. However, the details of the cross section limits similar to those presented by the ATLAS group for the $35 \mathrm{fb}^{-1}$ data used in obtaining our bounds, are not yet available for the $\mathcal{L}=1 \mathrm{fb}^{-1}$ data. We, therefore, use the following procedure.

From the ATLAS analysis in the jets $+\mathbb{E}_{T}$ channel based on $165 p b^{-1}$ of 2011 data [55] the limit on gluino mass is 500 for heavy $\operatorname{squarks}\left(m_{\tilde{q}}=1250\right)$. The CMS analysis in the same channel using $1.14 \mathrm{fb}^{-1}$ of 2011 data [53] puts a stronger limit on the gluino mass. For heavy squarks with average mass $1.5 \mathrm{TeV}(2 \mathrm{TeV})$, the lower limit on gluino mass is 570 (550). In the Lepton-Photon conference, 2011 [52] ATLAS results in the same channel was presented for $1.04 \mathrm{fb}^{-1}$ of 2011 data. For heavy squarks gluino mass below 600 are excluded.

Strictly speaking the above limits are not directly applicable in the LSG scenario. In this case a gluino decays via the cascade $\widetilde{g} \rightarrow \widetilde{t}_{1} t \rightarrow\left(b \widetilde{\chi}^{ \pm}\right)\left(b W^{ \pm}\right)$, yielding the signal whereas CMS and ATLAS consider gluino pair production in the limit where all squarks are heavy, so that each gluino decays via 3 body $\operatorname{modes}\left(q \bar{q} \widetilde{\chi}_{1}^{0}, q \bar{q} \widetilde{\chi}_{2}^{0}\right.$ or $\left.q q^{\prime} \widetilde{\chi}^{ \pm}\right)$. Jets are, therefore, likely to be softer in our case on the average. Hence the hard cuts on jet $p_{T}$ employed by the LHC collaborations are expected to give somewhat weaker limit on $m_{\widetilde{g}}$ in the LSG scenario. As a reasonable guess we have considered $m_{\widetilde{g}} \geq 550$ in the LSG scenario. Results for $m_{\widetilde{g}} \leq 500$ are already available in Section 2.

The ATLAS group has also updated their search for the $b l j E_{T}$ signal [54] in the LSG scenario. Using $1.03 \mathrm{fb}^{-1}$ data they have excluded gluino masses below $500-520 m_{\widetilde{t}_{1}}$ in the range 125 - 300. This limit, however, is also not applicable to the analysis in this paper based on a different chargino decay mode $\left(\operatorname{BR}\left(\widetilde{\chi}^{ \pm} \rightarrow \widetilde{\tau}_{1} \nu_{\tau}\right)=100 \%\right)$.

We present in Table 5 the observability of the signal in the phenomenological LSG model where $m_{\widetilde{t}_{1}}$ and $m_{\widetilde{g}}$ are unrelated parameter. This table illustrates the search prospect for

\footnotetext{
${ }^{9}$ The above comment is also applicable to the negative search in $b l j E_{T}$ channel by the ATLAS collaboration [54] briefly reviewed below.
} 


\begin{tabular}{|c|c||c|c|c||c|c|c|}
\hline \multicolumn{2}{|c||}{ Points } & \multicolumn{3}{|c||}{$\widetilde{t}_{1} \widetilde{t}_{1}^{*}$} & \multicolumn{3}{c|}{ LSG scenario } \\
\hline$m_{\widetilde{t}_{1}}$ & $m_{\widetilde{g}}$ & Cut Set 1 & Cut Set 2 & Cut Set 3 & Cut Set 1 & Cut Set 2 & Cut Set 3 \\
\hline \multirow{3}{*}{209} & 550 & & & & $394.4(9.4)$ & $285.8(9.3)$ & $17.4\left(4.3^{* *}\right)$ \\
& 700 & $362.9(8.7)$ & $272.3(8.9)$ & $4.5(1.1)$ & $378.3(9.0)$ & $275.4(9.0)$ & $10.3\left(2.5^{* *}\right)$ \\
& 850 & & & & $374.2(8.9)$ & $270.3(8.8)$ & $8.8(2.2)^{* *}$ \\
\hline \hline \multirow{3}{*}{250} & 550 & & & & $230.7(5.4)$ & $138.2\left(4.4^{* *}\right)$ & $16.3\left(4.0^{* *}\right)$ \\
& 650 & \multirow{2}{*}{$207.6(5.0)$} & \multirow{2}{*}{$136.0(4.4)$} & \multirow{2}{*}{$6.8(1.6)$} & $210.8(5.1)$ & $136.5\left(4.4^{* *}\right)$ & $10.2\left(2.5^{* *}\right)$ \\
& 750 & & & & $204.3\left(4.9^{* *}\right)$ & $132.1\left(4.2^{* *}\right)$ & $8.3(2.1)$ \\
\hline
\end{tabular}

Table 5: Notations, conventions and input parameters are the same as Table 2.

\begin{tabular}{|c|c|c||c|c|c|c|c||c||c|}
\hline$M_{3}$ & $\begin{array}{c}m_{0}(3) \\
(\widetilde{t})\end{array}$ & $\begin{array}{c}m_{0}(3) \\
(\widetilde{\tau})\end{array}$ & $m_{\widetilde{t}_{1}}$ & $m_{\widetilde{\chi}_{1}^{ \pm}}$ & $m_{\widetilde{\tau}_{1}}$ & $m_{\widetilde{\chi}_{1}^{0}}$ & $m_{\widetilde{g}}$ & $\begin{array}{c}\text { S1 } \\
(\text { Cut Set 1) }\end{array}$ & $\begin{array}{c}\text { S3 } \\
\text { (Cut Set 3) }\end{array}$ \\
\hline \hline 210 & 365 & 112 & 229 & 186 & 106 & 97 & 567 & $228.7(5.5)$ & $16.7\left(4.1^{* *}\right)$ \\
& 420 & 114 & 283 & 188 & 105 & 98 & 569 & $101.9\left(2.4^{* *}\right)$ & $12.2\left(3.0^{* *}\right)$ \\
\hline 225 & 330 & 112 & 231 & 185 & 106 & 97 & 600 & $218.8(5.2)$ & $13.9\left(3.5^{* *}\right)$ \\
\hline 250 & 250 & 111 & 235 & 184 & 106 & 97 & 657 & $201.3\left(4.8^{* *}\right)$ & $9.8\left(2.4^{* *}\right)$ \\
\hline
\end{tabular}

Table 6: Notations, conventions and input parameters are the same as in Table 4.

$m_{\widetilde{g}} \geq 550$. The notations, input parameters consistent with a LMNDM scenario and cuts are the same as in Table 2. The corresponding results for the scenario with nonuniversal boundary conditions at the GUT scale (see Section 2) are presented in Table 6. It may also be noted that if $\mathcal{L}=10 \mathrm{fb}^{-1}$ is accumulated in the LHC $7 \mathrm{TeV}$ run then mass reach in this channel will be considerably improved. For example if $m_{\widetilde{g}}=550$ then $m_{\widetilde{t}_{1}} \sim 315$ can be probed with Cut Set 1.

The new data from LHC based on $\mathcal{L}=1 \mathrm{fb}^{-1}$, however, strongly disfavor the LMNDM scenario in the mSUGRA model irrespective of the experimental uncertainties. We superimpose on Figure 2 the region excluded by the CMS collaboration [53]. It is readily seen that for low $m_{0}$, neutralino - stau coannihilation is the only viable mechanism for DM relic density production provided $m_{\widetilde{\chi}_{1}^{0}} \geq 215$. 
Even if the issue of neutralino dark matter is set aside, the $b \tau j E_{T}$ is unobservable in the mSUGRA model even for $\mathcal{L}=5 \mathrm{fb}^{-1}$ (see the recent CMS constraint reproduced in figure 2). The $\operatorname{blj} E_{T}$ signal is observable in a small corner of the parameter space thanks to the experimental uncertainties as reflected by the CMS expected limit $\pm 1 \sigma$ band.

\section{Summary and conclusions}

The data from the on going experiments at the LHC has put a question mark on the viability of a substantial region of the mSUGRA parameter space corresponding to low mass sparticles. This leads to a tension between the data and the low mass neutralino dark matter (LMNDM) scenario where neutralino annihilation and/ or neutralino - slepton coannihilation can produce DM relic densities consistent with the WMAP data. Moreover, large regions of the parameter space sensitive to the ongoing experiments for direct DM search or searches in the near future are also under pressure.

However, the LHC experiments are primarily sensitive to the masses of the strongly interacting sparticles - the squarks and the gluinos. In contrast in typical LMNDM scenarios the relic density may depend entirely on the properties of the sparticles in the electroweak sector. Thus the above tension is an artifact of the model dependent correlations among the soft breaking masses in the strong and the electroweak sectors of mSUGRA. Any model in which the masses in the two sectors are independent parameters could be free from this tension.

In view of this we propose a few generic models which are unconstrained or mildly constrained by the LHC data. These models are generic in the sense that their acceptability depends on certain mass hierarchies in the strong sector and not on specific mass values. The electroweak sector is assumed to be independent of the strong sector. In fact we only assume that the LSP is bino like, $\tan \beta$ has intermediate values and all parameters in the EW sector are consistent with the corresponding LEP limits provided such limits are not based on mSUGRA dependent assumptions.

In the first phenomenological model under consideration all strongly interacting sparticles except for the lighter top squark are assumed to be beyond the reach of the experiments at LHC-7 TeV (see Section 2). This squark and the electroweak sparticles are the only sources of SUSY signals. For simplicity we assume that the masses of the relatively light 
sparticles are correlated as in mSUGRA. Our conclusions will obviously hold in a more general framework. In this case the trilinear soft breaking parameter $\left(A_{0}\right)$ must be non-zero. This ensures consistency with the bound on the Higgs mass from LEP [15]. Moreover, for a given $m_{0}$, LSP- $\widetilde{\tau}_{1}$ coannihilation may produce the observed relic density for values of $m_{1 / 2}$ significantly lower than that for $A_{0}=0$ [15]. This also facilitates the LMNDM scenario.

The conventional SUSY signals with hard cuts on $\mathbb{E}_{T}$ or $m_{\text {eff }}$ are not viable in this model. However, a fairly large region of the parameter space can be probed by the $b \tau j E_{T}$ signal (see Table 1 and the reach plot Fig. 1) using the search strategy sketched in this paper. It is estimated that $m_{\widetilde{t}_{1}} \leq 235(280)$ can be probed with $\mathcal{L}=1(5) \mathrm{fb}^{-1}$. A part of this parameter space is consistent with the WMAP data where the DM relic density is produced by bulk annihilation as shown in Fig 1. However, this result is subject to certain simplifying assumptions (see Section 2 paragraph 4). In an unconstrained MSSM the signal will be compatible with a much larger parameter space consistent with the LMNDM scenario.

The complementary signal blj $E_{T}$ signal proposed in [28] may be useful if the charginos do not dominantly decay into tau rich final states. The possibility that signals from stop pair production only are already buried in the LHC data is still open.

We next focus on a model (the LSG scenario)in which only $\widetilde{t}_{1}$ and $\widetilde{g}$ but no other strongly interacting sparticle, are within the striking range of the ongoing LHC experiments. As before the EW sector is assumed to be independent of the strong sector and is taken to be consistent with the WMAP data. This model is only mildly constrained by the LHC data for $\mathcal{L}=35 \mathrm{pb}^{-1}$ (see Table 2 for some representative examples). The $b \tau j E_{T}$ signal can probe a fairly large region of the parameter space (Table 2). Moreover, using additional cuts the stop induced events can be separated from the gluino induced ones (see Table 2). The sample thus separated can be used for reconstruction of the properties of $\tilde{t}_{1}$.

The very recent data for $\mathcal{L}=1 \mathrm{fb}^{-1}$, however, make the lower limit on $m_{\widetilde{g}}$ more stringent $\left(m_{\widetilde{g}} \geq 550\right)$ for heavy squarks (see Section 4$)$. Thus the contribution of gluino pair production to the signal reduce significantly. Still the stop induced and gluino induced contributions can be separated by suitable cuts as illustrated in (Table 5).

The LSG scenario can be realized in a mSUGRA type model with nonuniversal boundary conditions at $M_{G}$ (see Table 4 for sample results for $\mathcal{L}=35 \mathrm{pb}^{-1}$ ). The electroweak sector chosen is consistent with a LMNDM scenario. The updated results corresponding to $\mathcal{L}=1$ $\mathrm{fb}^{-1}$ data are presented in Table 6 . 
Finally we look into the $b \tau j E_{T}$ signal in the mSUGRA model (Section 3, Figure 2). We also revisit the $b l j \mathbb{E}_{T}$ signal in case it gives a better reach in some parameter space. So far as the $\mathcal{L}=35 \mathrm{pb}^{-1}$ data is concerned both the above signals and the LMNDM scenario cannot be strictly ruled out due to the uncertainties in the data with low statistics ( the ATLAS constraints are reproduced in Fig. 2 for ready reference). However, the more recent data for $\mathcal{L}=1 \mathrm{fb}^{-1}$ strongly disfavors both the signals and the LMNDM scenario (see the recent CMS constraints superimposed on Fig 2).

The latest LHC data also disfavors a large parameter space in mSUGRA with low mass neutralinos in mSUGRA sensitive to the direct DM search by the XENON100 experiment. Alternative models for LMNDM may, therefore, call for more attention.

In this paper we have not considered constraints from flavour physics like the flavour violating decays of the B-hadrons. Strictly speaking these constraints are sensitive to the additional assumption that the quark and the squark mass matrices are aligned in the flavour space so that the same matrix as the CKM matrix also operate in the squark sector. This assumption of minimum flavour violation fails even if there are small off-diagonal elements of the squark mass matrix at the GUT scale. On the other hand such small element does not affect processes like neutralino annihilation and squark-gluino production and decay. In fact, it has been explicitly shown that such small mixings at the GUT scale can significantly weaken the constraints from flavour physics [56]. Moreover a comprehensive analysis of all possible constraints on a SUSY model is beyond the scope of this paper.

We conclude that any model whose strong sector is not sensitive to the current LHC data, may be consistent with a LMNDM scenario satisfying the WMAP constraints thanks to the properties of the EW sector, provided model dependent correlations among the parameters of the strong and the electroweak sectors, as in mSUGRA, are given up. Thus there is no serious conflict between the LMNDM scenario and the LHC data.

\section{References}

[1] G. Aad et al ATLAS Collaboration, Phys. Rev. Lett. 106, 131802 (2011); G. Aad et al ATLAS Collaboration, Phys. Lett. B 701, 186 (2011); ATLAS Collaboration, Phys. Lett. B 701, 398 (2011). 
[2] V. Khachatryan et al CMS Collaboration, Phys. Lett. B 698, 196 (2011); S. Chatrchyan et al CMS Collaboration, J. High Energy Phys. 1106, 093 (2011); S. Chatrchyan et al CMS Collaboration, arXiv: 1103.0953; S. Chatrchyan et al CMS Collaboration, arXiv: 1104.1617; S. Chatrchyan et al CMS Collaboration, arXiv: 1106.3272.

[3] For the latest limits on the sparticle masses from LEP experiments: see,e.g., http://lepsusy.web.cern.ch/lepsusy/.

[4] CDF collaboration, T. Aaltonen et al., Phys. Rev. Lett. 102, 121801 (2009); D0 collaboration (V. M. Abazov et al), Phys. Lett. B 660, 449 (2008).

[5] A. H. Chamseddine, R. Arnowitt and P. Nath, Phys. Rev. Lett. 49, 970 (1982); R. Barbieri, S. Ferrara and C. A. Savoy, Phys. Lett. B 119, 343 (1982); L. J. Hall, J. Lykken and S. Weinberg, Phys. Rev. D 27, 2359 (1983); P. Nath, R. Arnowitt and A. H. Chamseddine, Nucl. Phys. B 227, 121 (1983); N. Ohta, Prog. Theor. Phys. 70, 542 (1983).

[6] L. E. Ibanez and G. G. Ross, Phys. Lett. B 110, 215 (1982); K. Inoue, A. Kakuto, H. Komatsu and S. Takeshita, Prog. Theor. Phys. 68, 927 (1982) [Erratum-ibid. 70, 330 (1983)]; J. R. Ellis, J. S. Hagelin, D. V. Nanopoulos and K. Tamvakis, Phys. Lett. B 125, 275 (1983); L. Alvarez-Gaume, J. Polchinski and M. B.Wise, Nucl. Phys. B 221, 495 (1983).

[7] D. Feldman, Z. Liu and P. Nath, Phys. Rev. D 81, 095009 (2010); H. Baer, S. Kraml, A. Lessa and S. Sekmen, J. High Energy Phys. 02, 055 (2010); H. K. Dreiner, M. Kramer, J. M. Lindert and B. O'Leary, J. High Energy Phys. 04, 109 (2010); H. Baer, V. Barger, A. Lessa, and X.Tata, J. High Energy Phys. 06, 102 (2011); N. Chen, D. Feldman, Z. Liu, P. Nath and G. Peim, Phys. Rev. D 83, 035005 (2011); B. Altunkaynak, M. Holmes, P. Nath, B. D. Nelson and G. Peim, Phys. Rev. D 82, 115001 (2010); B. Mukhopadhyaya and S. Mukhopadhyay, Phys. Rev. D 82, 031501 (2010); S. Akula et al, Phys. Lett. B 699, 377 (2011); P. Bechtle et al, arXiv:1105.5398; M. Guchait and D. Sengupta,Phys. Rev. D 84, 055010 (2011).

[8] Nabanita Bhattacharyya, Amitava Datta and Sujoy Poddar, Phys. Rev. D 82, 035003 (2010). 
[9] G. Bertone, D. Hooper and J. Silk, Phys. Rept. 405, 279 (2005), W. L. Freedman and M. S. Turner, Rev. Mod. Phys. 75, 1433 (2003), L. Roszkowski, Pramana 62, 389 (2004).

[10] H. Baer and X. Tata in Physics at the Large Hadron Collider, Indian National Science Academy, A Platinum Jubilee Special Issue (Eds. Amitava Datta, B. Mukhopadhyaya and A. Raychaudhuri; Springer, 2009).

[11] D. N. Spergel et al., Astrophys. J. Suppl. 170, 377 (2007).

[12] H. Glodberg,Phys. Rev. Lett. 50, 1419 (1983); J. Ellis, J. Hagelin, D. V. Nanopoulos and M. Srednicki,Phys. Lett. B 127, 233 (1983); M. Drees and M. Nojiri, Phys. Rev. D 47, 376 (1993); H. Baer and M. Brhilik,Phys. Rev. D 53, 597 (1996); V. Barger and C. Kao,Phys. Rev. D 57, 3131 (1998).

[13] Zones in the mSUGRA parameter space consistent with WMAP data have been identified by many groups. See, e.g., J. R. Ellis, K. A. Olive, Y. Santoso and V. Spanos,Phys. Lett. B 565, 176 (2003); H. Baer et al,J. High Energy Phys. 0306, 054 (2003); U. Chattopadhyay, A. Corsetti and P. Nath,Phys. Rev. D 68, 035005 (2003); A. Djouadi, M. Drees and J. L. Kneur, J. High Energy Phys. 0603, 033 (2006).

[14] J. R. Ellis, T. Falk and K. A. Olive, Phys. Lett. B 444, 367 (1998); J. R. Ellis, T. Falk, K. A. Olive and M. Srednicki, Astropart. Phys. 13, 181 (2000) [Erratumibid. 15, 413 (2001)]; M. E. Gomez, G. Lazarides and C. Pallis,Phys. Rev. D 61, 123512 (2000); A. Lahanas, D. V. Nanopoulos and V. Spanos, Phys. Rev. D 62, 023515 (2000); R. Arnowitt, B. Dutta and Y. Santoso, Nucl. Phys. B606, 59(2001); T. Nihei, L. Roszkowski and R. Ruiz de Austri, J. High Energy Phys. 0207, 024 (2002); V. A. Bednyakov, H. V. Klapdor-Kleingrothaus and V. Gronewold, Phys. Rev. D 66, 115005 (2002).

[15] Utpal Chattapadhyay, Debottam Das, Amitava Datta and Sujoy Poddar,Phys. Rev. D 76, 055008 (2007).

[16] S. Akula et al, arXiv:1103.5061.

[17] For a review see, e.g, R. J. Gaitskell, Annu. Rev. Nucl. Part Sci., 54,315,2004 and references there in. 
[18] E. Aprile et al (XENON100 Collaboration), arXiv:1104.2549; arXiv:1103.4160;Phys. Rev. Lett. 105, 131302 (2010).

[19] Z. Ahmed et al,Phys. Rev. Lett. 102, 011301 (2009);Science 327, 1619(2010).

[20] S. Profumo, arXiv:1105.5162; O. Buchmueller et al, arXiv:1106.2529.

[21] M. Drees and C-L.Shan, J. Cosmol. Astropart. Phys. 06, 011 (2007); O.Mena, S. Palomarez-Ruiz and S. Pascoli,Phys. Lett. B 664, 92 (2008); A. M. Green,J. Cosmol. Astropart. Phys., 0807,005 (2008); C-L. Shan, New.J.Phys.11,105013(2009); N. Bernal, A. Goudelis, Y. Mambrini and C. Munoz,J. Cosmol. Astropart. Phys. 0901,046(2009). J. Billard, F. Mayetand D. Santos,Phys. Rev. D 83, 075002 (2011).

[22] J. A. Conley, H. K. Dreiner and P. Wienemann, Phys. Rev. D 83, 05501 (2011).

[23] D. Feldman, Z. Liu and P. Nath, Phys. Rev. D 81, 117701 (2010); E. Kuflik, A. Pierce and K. M. Zurek, Phys. Rev. D 81, 111701 (2010); C. Boehm, J. Silk and T. Ensslin, arXiv:1008.5175[astro-ph.GA].

[24] R. Barate et al [LEP Working Group for Higgs boson searches], Phys. Lett. B 565, 61 (2003).

[25] See, e.g., Fig. 13.5 in CMS physics Technical Design Report, Vol-II (Eds. A. De Roeck et al), J. Phys. G 34, 995 (2007).

[26] L. S. Stark, P. Hafliger, A. Biland and F. Pauss, J. High Energy Phys. 0508, 059 (2005).

[27] Nabanita Bhattacharyya, Amitava Datta and Sujoy Poddar, Phys. Rev. D 78, 075030 (2008).

[28] Nabanita Bhattacharyya, Arghya Choudhury and Amitava Datta, Phys. Rev. D 83, 115025 (2011).

[29] D. Feldman, Z. Liu and P. Nath, Phys. Rev. Lett. 99, 251802 (2007); J. High Energy Phys. 0804, 054 (2008); C.F. Berger, J.S. Gainer, J.L. Hewett and T.G. Rizzo, J. High Energy Phys. 02, 023 (2009); P. Konar, K. T. Matchev, M. Park, G. K. Sarangi Phys. Rev. Lett. 105, 221801 (2010). 
[30] D. Hooper and T. Plehn, Phys. Lett. B 562, 18 (2003); G.Belanger, F. Boudjema, A. Phukov and S. Rosier-Lees, hep-ph/0212227; A. Bottino, F. Donato, N. Forengo and S. Scopel,Phys. Rev. D 68, 043506 (2003); G. Belanger et al, J. High Energy Phys. 03, 212 (2004); J. Lee and S. Scopel, Phys. Rev. D 75, 015005 (2007); S. Profumo,Phys. Rev. D 78, 023507 ( 2008); H. K. Dreiner et al, Eur. Phys. J. C 62, 547 (2009).

[31] J. M. Frere, D. R. T. Jones and S. Raby, Nucl. Phys. B 222, 11 (1983).

[32] For recent values of $m_{t}$, see, e.g., F. Margaroli (CDF collaboration), arXiv:1105.5183.

[33] A. Djouadi, J. L. Kneur and G. Moultaka, Comp. Phys. Comm. 176, 426 (2007).

[34] M. Muhlleitner, A. Djouadi and Y. Mambrini, Comp. Phys. Comm. 168, 46 (2005).

[35] T. Sjostrand, P. Eden, C. Friberg, L. Lonnblad, G. Miu, S. Mrenna and E. Norrbin, Comp. Phys. Comm. 135, 238 (2001); For a more recent version see, J. High Energy Phys. 0605, 026 (2006).

[36] See, e.g. , A. Pukhov, CalcHEP-a package for evaluation of Feynman diagrams and integration over multi-particle phase space (hep-ph/9908288). For the more recent versions see: http://www.ifh.de/pukhov/calchep.html.

[37] H. L. Lai et al. [CTEQ Collaboration], Eur. Phys. J. C 12, 375 (2000); P. M. Nadolsky et al. [CTEQ Collaboration], Phys. Rev. D 78, 013004 (2008). See also, http://www.phys.psu.edu/cteq.

[38] M. Mangano et al. , J. High Energy Phys. 0307, 001 (003).

[39] W. Beenakker, R. Hoepker and M.Spira, arXiv: hep-ph/9611232

[40] S. K. Soni and H. A. Weldon, Phys. Lett. B 126, 215 (1983); M. Drees, Phys. Lett. B 181, 279 (1986); Y. Kawamura, H. Murayama and M.Yamaguchi, Phys. Rev. D 51, 1337 (1995); A. Gouvea, A. Friedland and H. Murayama, Phys. Rev. D 59, 095008 (1999); S. Profumo, Phys. Rev. D 68, 015006 (2003).

[41] Amitava Datta, M. Guchait and N. Parua, Phys. Lett. B 395, 54 (1997); P. Nath and R. Arnowitt, Phys. Rev. D 56, 2820 (1997). Amitava Datta, Aseshkrishna Datta, M. K. Parida, Phys. Lett. B 431, 347 (1998); Amitava Datta, Aseshkrishna Datta, M. 
Drees and D. P. Roy, Phys. Rev. D 61, 055003 (2000); S. Bhattacharyya, AseshKrishna Datta and B. Mukhopadhyaya, Phys. Rev. D 78, 035011 (2008); S. Bhattacharyya, U. Chattopadhyay, D. Choudhury, D. Das and B. Mukhopadhyaya, Phys. Rev. D 81, 075009 (2010); S. Bhattacharya, S. Biswas, B. Mukhopadhyaya and M. M. Nojiri, arXiv: 1105.3097 .

[42] R. Arnowitt, A. H. Chamseddine and P. Nath, Problems in Unification and Supergravity, La Jolla Institute (1983) (see http://www.osti.gov/bridge/servlets/purl/5986323fY7neR/5986323.pdf); J. R. Ellis, K. Enqvist, D. V. Nanopoulos and K. Tamvakis, Phys. Lett. B 155, 381 (1985); M. Drees, Phys. Lett. B 158, 409 (1985); A. Corsetti and P. Nath, Phys. Rev. D 64, 125010 (2001); S. P. Martin, Phys. Rev. D 79, 095019 (2009).

[43] G. Anderson, H. Baer, C. Chen and X. Tata, Phys. Rev. D 61, 095005 (2000); A. Corsetti and P. Nath,Phys. Rev. D 64, 125010 (2001);U. Chattopadhyay and P. Nath,Phys. Rev. D 65, 07509 (2002); S. Bhattacharyya, AK Datta, and B. Mukhopadhyaya, J. High Energy Phys. 10, 080 (2007); S. Bhattacharya and J. Chakrabortty, Phys. Rev. D 81, 015007 (2010).

[44] M. Drees Phys. Rev. D 33, 1468 (1986); For reviews and more references see, e.g., J. Amundson et al., in Report of the Supersymmetry Theory Working Group, ECONF C960625:SUP106,1996 (arXiv:hep-ph/9609374v2); G. Anderson, C. H. Chen, J. F. Gunion, J. Lykken, T. Moroi and Y. Yamada, arXiv:hep-ph/9609457.

[45] H. Baer, A. Mustafayev, E. Park and X. Tata, J. High Energy Phys. 0805, 058 (2008); H. Baer, M. A. Diaz, P. Quintana and X. Tata, J. High Energy Phys. 0004, 016 (2000); H. Baer, S. Kraml, A. Lessa, S. Sekmen and Xerxes Tata, J. High Energy Phys. 1010, 018 (2010).

[46] V. Berezinski et al Astropart. Phys. 5333 (1996); A. Bottino, F. Donaldo, N. Forengo and S. Scopel,Phys. Rev. D 59, 095004 (1999); J. R. Ellis, K. Olive and Y. Santoso, Nucl. Phys. B 652, 259 (2003);J. Ellis, S. F. King and J. R. Roberts ,arXiv:0711.2741. See also [41].

[47] N. Bhattacharyya and Amitava Datta,Phys. Rev. D 80, 055016 (2009). 
[48] For recent discussions on slepton pair production at hadron colliders and references to earlier works see: G. Bozzi, B. Fucks and M. Klasen, Phys. Rev. D 74, 015001 (2006);Nucl. Phys. B 777, 157 (2007); Nucl. Phys. B 794, 46 (2008); F. Borzumati and K. Hagiwara, J. High Energy Phys. 03, 103 (2011).

[49] H. Baer, K. Hagiwara and X. Tata, Phys. Rev. D 35, 1598 (1987); R. Arnowitt and P. Nath, Mod. Phys. Lett. A 2, 331 (1987); H. Baer and X. Tata, Phys. Rev. D 47, 2739 (1993); H. Baer, C. Kao and X. Tata, Phys. Rev. D 48, 5175 (1993); S. Mrenna, G. Kane, G. D. Kribs and J. D. Wells, Phys. Rev. D 53, 1168 (1996); Zack Sullivan, Edmond L. Berger, Phys. Rev. D 78, 034030 (2008).

[50] see [25], Section 13.14

[51] see [25], Section 13.15

[52] Talk by Henri Bachacou on behalf of the ATLAS and CMS Collaborations at LeptonPhoton 2011,Mumbai. See, http://www.tifr.res.in/ lp11/

[53] CMS Collaboration, arXiv 1109.2352 [hep-ex].

[54] ATLAS Collaboration, ATLAS-CONF-2011-130 (2011).

[55] ATLAS Collaboration, ATLAS-CONF-2011-086 (2011).

[56] K. Okumura and L. Roszkowski Phys. Rev. Lett. 92, 161801 (2004) 